\title{
Using latent class and quantum models to value equity in health care: a tale of 2 stories
}

\author{
ABSTRACT \\ Cost-effectiveness analysis (CEA) with qual- \\ ity-adjusted life-year (QALY) was introduced \\ to address health equity concerns in value \\ assessment. However, QALY fails to capture \\ patient preference. Stated preference meth- \\ ods (eg, discrete choice experiment [DCE]) \\ have been increasingly used to incorporate \\ patient preference into the value assessment \\ framework in health care. Still, ones with a \\ moral dimension such as health equity do \\ not exist. \\ The objective of this paper was to \\ describe 2 stated preference approaches \\ that can empirically value health equity.
}

First, the decision-maker perceptions of the prevalence of equity dimensions in DCE choice tasks are identified. A latent class model based on random utility theory is proposed to derive the value of equity from the decision makers with different perceptions of the prevalence of equity dimensions. Second, equity attributes are incorporated in DCE choice tasks, and a quantum choice model, which can capture stochasticity during the decision process in the mind of the decision makers, is used to value the equity. These approaches will improve existing value assessment methods to address health equity adequately.

\section{To love another person is to see the face of God -Victor Hugo, Les Misérables.}

Despite the increasing interest in developing the assessments of novel value elements identified by the International Society of Pharmacoeconomics and Outcomes Research (ISPOR) Special Task Force, ${ }^{1}$ relatively few attempts have been made to value equity. None of the US value assessment frameworks directly defined equity or proposed an empirical measure of the equity. Only some potential elements (eg, unmet need) were considered. $^{2}$

In 2017, cost-effectiveness analysis (CEA) with quality-adjusted life-year (QALY) was introduced to address health equity concerns in value assessment. ${ }^{3}$ Primarily, health economists used 2 approaches, including equity impact analysis and equity trade-off analysis, to address these concerns. ${ }^{3}$ The equity impact analysis quantifies the distribution of costs and effects by equity relevant variables. Two examples of the equity impact analysis include extended CEA and distributional CEA.

On the other hand, the equity trade-off analysis quantifies tradeoffs between improving total health and other equity objectives. Two main approaches to equity trade-off analysis include equity constraint analysis and equity weighting analysis, which are counting the cost of equity and valuing the importance of equity, respectively. Similar to the equity impact analysis, CEA with QALYs has been used to capture the health loss associated with choosing a more equitable option in the equity constraint

\author{
Author affiliation \\ Surachat Ngorsuraches, PhD, Department \\ of Health Outcomes Research \& Policy, \\ Harrison School of Pharmacy, Auburn \\ University, Auburn, AL. \\ AUTHOR CORRESPONDENCE: \\ Surachat Ngorsuraches, 334.844.8357; \\ surachat@auburn.edu \\ J Manag Care Spec Pharm \\ 2021;27(9-a):S12-S16 \\ Copyright $\odot 2021$, Academy of Managed \\ Care Pharmacy. All rights reserved.
}

analysis, and QALY is used as a common metric in the equity-weighting analysis.

Despite the wide use of the QALY metric in value assessment, it remains controversial because it is a single-dimensional and generic health measure. Furthermore, CEA with QALYs fails to capture patient preference and do not address the heterogeneity of patient preference. Although stated preference methods (eg, discrete choice experiment [DCE] and best-worst scaling [BWS]) have been increasingly used to incorporate patient preference into the value assessment framework in health care, the ones with a moral dimension, such as health equity, do not exist. There is, therefore, a critical need to improve existing methods that inadequately address health equity issues. Literature-suggested methods 


\section{FIGURE 1 Using a Latent Class Model to Value Equity}

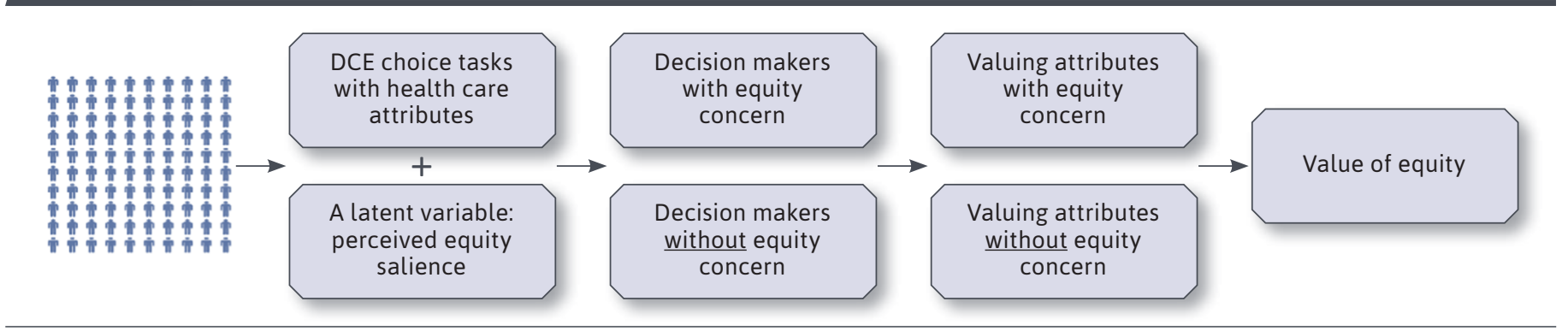

$D C E=$ discrete choice experiment.

\section{FIGURE 2 Conceptual Model of an Individual's Moral Choice Behavior ${ }^{a}$}

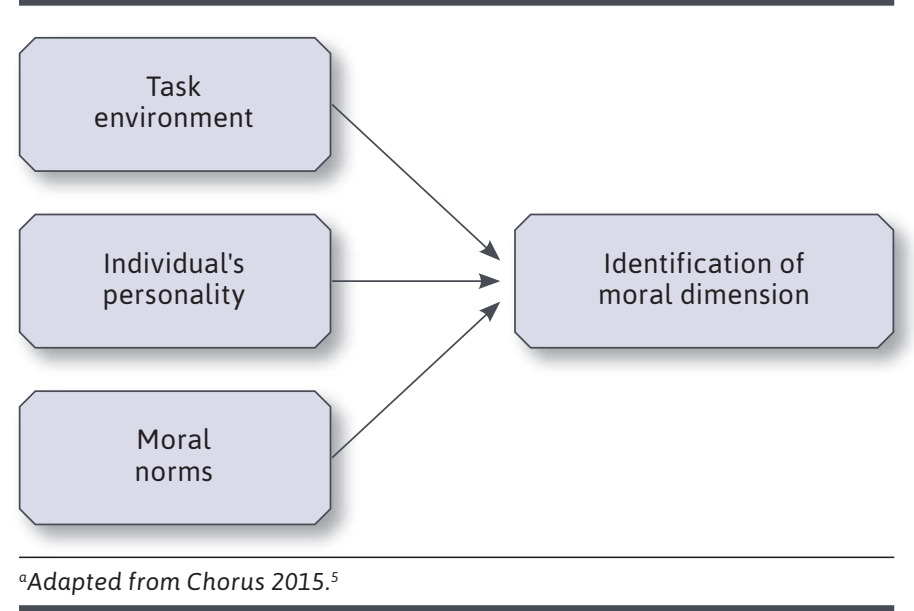

from various disciplines should be harnessed to rigorously translate people's considered preferences to address health equity issues and to give guidance to health care decision makers. ${ }^{4}$

The objective of this paper is to describe 2 approaches that empirically value health equity and capture decisionmaker preferences to contribute to value assessment under the realization of health equity. These approaches can be used to ensure the systematic consideration of health equity in decision making, and they will also determine how equity enters the preferences of decision makers, indicated as another challenge by ISPOR Special Task Force. ${ }^{1}$

\section{Proposed Approaches}

Only a few stated preference studies, which explicitly acknowledged and explored the moral dimensions of choice behaviors, exist. ${ }^{4,5}$ One of the major challenges is that sometimes the moral choice is obvious, and, at other times, it is more latent or implicit. ${ }^{4}$ A moral dimension may be considered to some extent in the choice situations of these stated preference studies; however, these studies do not explicitly consider the moral dimension of the decision. This paper proposed to use 2 approaches previously developed and applied to examine the moral dimension in the transportation field to overcome this challenge and address equity concerns in health care. ${ }^{4,6}$

\section{FIRST APPROACH}

This approach uses DCE with a latent class model to derive the value of equity. Figure 1 shows the steps of this approach. In general, a DCE describes various choice tasks of study treatment or health care service by its attributes (eg, efficacy, side effects, and costs). To engage patients in this approach, these attributes should be obtained from patient experiences. Each choice task contains various hypothetical alternatives with different attributes and levels, randomly combined by a rigorous method of DCE study design. Participants (eg, patients, providers, policymakers, and taxpayers) will be asked to choose 1 alternative that they prefer from each choice set. To value the equity and capture decision-maker preferences to contribute to value assessment under the consideration of equity, an individual's perception of the prevalence of equity dimensions in the choice tasks needs to be determined. Subsequently, responses to the DCE choice tasks from participants with different perceptions of the prevalence of equity dimensions are obtained. 


\begin{tabular}{l|c|c}
\hline \multicolumn{1}{|c}{ TABLE 1} & An Example of Results From the Latent Class Approach \\
\cline { 2 - 3 } & Class with equity salience & Class without equity salience \\
\hline $\begin{array}{l}\text { Marginal willingness to pay } \\
\text { for health gain }\end{array}$ & $\$ X$ & $\$ Y$ \\
\hline
\end{tabular}

A literature review from the fields of economics and psychology was used to develop the conceptual model of an individual's moral choice behaviors and suggested to create a latent variable, "perceived moral salience," that can be used to reflect the individual's identification of moral dimension. ${ }^{4}$ The model identifies the task environment, the individual's personality, and moral norms as factors influencing the individual's identification of moral dimension (Figure 2). This model indicates that people can simply adjust their decision making when they encounter or identify a task environment with a moral dimension. Morality should at least to some extent be considered as a personality trait. As a result, different individuals from the same culture behave differently when encountered with the same moral situation. Many people prefer to stick with norms, even if these norms conflict with their personal preferences.

Therefore, this paper proposes to construct the perceived equity salience variable as a function of task environment (eg, the presence or absence of explicit verbal cues about health equity), individual's personality (eg, social value orientation), and prevailing moral norm of equity to determine the individual's identification of a given choice situation as having an equity dimension or not. Based on random utility theory (RUT), the perceived equity salience variable helps develop a latent class model. Individuals with different levels of the perceived equity salience variable are assigned with different probabilities to "decision makers with equity concern" and "decision makers without equity concern" classes. These 2 classes are specified to differ in terms of preference weights or values for study attributes, based on the marginal rates of substitution between each attribute and cost. In other words, 2 value sets of treatment or health care service attributes determined with and without equity concerns are obtained. These 2 value sets can be compared to reflect the value of equity.

Preferences for the attributes under the realization of equity can be captured from the class of decision makers with equity concerns. For example, this approach can estimate social value for health gains from treatments for an underrepresented population (which can also be more specific populations). A DCE survey is designed to include health gain and cost as study attributes. Taxpayers are asked to respond to the survey. Marginal willingness-to-pay (WTP) for health gains can be calculated. Table 1 shows an example of the results. The marginal WTPs for health gains from the perspective of taxpayers with and without perceived equity salience are SX and \$Y, respectively. Assuming \$X and $\$ Y$ to include the implicit value of equity, the difference between these amounts (\$X and \$Y) reflects the value of equity for 1 unit of health gain.

This approach can also examine how equity enters the participant preferences identified by the ISPOR Special Task Force as an unclear issue. ${ }^{1}$ For instance, the latent variable perceived equity salience could be modified to capture whether the participants focus equity on overall well-being or specifically on equity in health.

\section{SECOND APPROACH}

This approach uses DCE that incorporates equity attributes for individual alternatives in choice tasks to derive the value of equity. Figure 3 shows the steps of this approach. Participants (eg, patients, providers, policymakers, and taxpayers) are asked to complete 2 sets of DCE choice tasks based on an individual's preference. The first set involves trade-offs among treatment or health care attributes, including cost. Traditional choice models (eg, mixed logit model) can be used for this first set of choice tasks. The second set also includes equity attributes (eg, increased benefits and decreased risks for others in study treatment or health care) meaning that the participants need to make choices with equity concerns. An experimental study indicated that people not only preferred a resource allocation rule that most benefited them but also judged it to be fairer and more moral. ${ }^{7}$ In this given choice context, people may evaluate the choice tasks differently because of equity attributes. Their decisions would have changed depending on the acceptance or dismissal of the equity components. The study also suggested people could change their views about equity in a matter of minutes as they learned where their interests lay. Therefore, the stochasticity during the decision process in the mind of the individual decision maker needs to be captured to reflect behavioral realism.

RUT, which follows the classic theories of probability and has dominated the choice modeling field for decades and has been criticized as 


\section{FIGURE 3 Using the Quantum Choice Model to Value Equity}

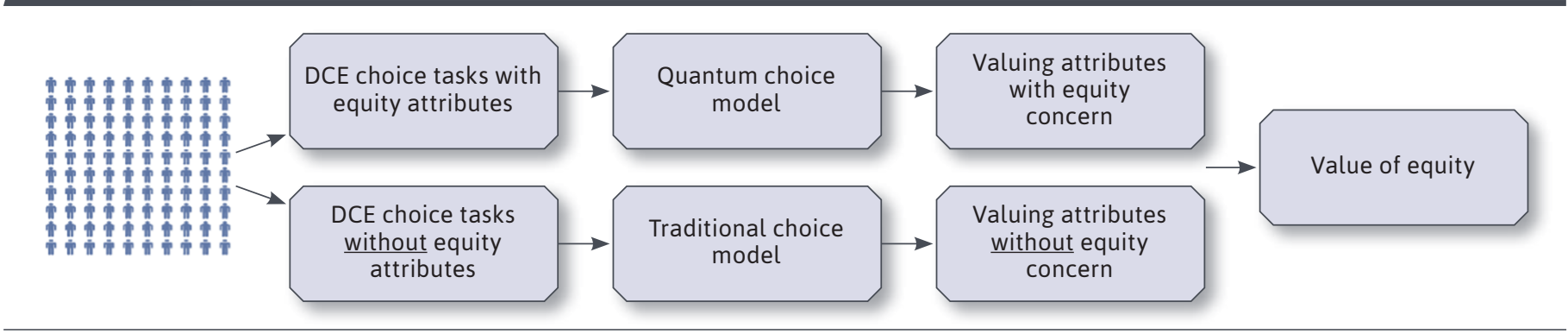

$D C E=$ discrete choice experiment.

\section{TABLE 2 An Example of the Results From the Quantum Approach}

\begin{tabular}{|c|c|c|c|c|c|}
\hline \multicolumn{6}{|c|}{ Observed share of an alternative under each choice task } \\
\hline \multirow[b]{2}{*}{ Choice task } & \multicolumn{4}{|c|}{ Attribute changes } & \multirow{2}{*}{$\begin{array}{c}\text { Observed share of } \\
\text { alternative A, \% }\end{array}$} \\
\hline & Own health gain & Own cost & Others' health gain & Others' costs & \\
\hline 1 & Included & Included & - & - & 70 \\
\hline 2 & Included & Included & - & - & 71 \\
\hline 3 & Included & Included & Included & Included & 80 \\
\hline 4 & Included & Included & Included & Included & 85 \\
\hline 5 & Included & Included & Included & Included & 82 \\
\hline \multicolumn{6}{|c|}{ Results from a basic choice model and a quantum choice model with rotation } \\
\hline & & \multicolumn{4}{|c|}{ Average probability of chosen alternative $A$} \\
\hline \multicolumn{2}{|c|}{ Choice task } & \multicolumn{2}{|c|}{ Basic model, \% } & \multicolumn{2}{|c|}{ Quantum model with rotation, \% } \\
\hline \multicolumn{2}{|c|}{ Choice tasks without others' health gain and cost } & \multicolumn{2}{|c|}{83} & \multicolumn{2}{|c|}{70} \\
\hline \multicolumn{2}{|c|}{ Choice tasks with others' health gain and cost } & \multicolumn{2}{|c|}{80} & \multicolumn{2}{|c|}{83} \\
\hline
\end{tabular}

being inadequate in explaining moral choice behavior. It can be complex because decision makers may choose an alternative based not only on more attractive concrete attributes of the alternative but also how they believe the alternative to be an overall morally contentious option. ${ }^{6,8}$ Recently, quantum probability theory has been introduced in cognitive psychology. ${ }^{9}$ One of the key differences of the quantum theory from the classic theories of probability is that the distributivity law of "and" and "or" propositions$\mathrm{A} \Lambda(\mathrm{B} \vee \mathrm{C})=(\mathrm{A} \Lambda \mathrm{B}) \mathrm{v}(\mathrm{A} \Lambda \mathrm{C})-$ does not need to hold. ${ }^{9}$ This difference resulted in the creation of a new theory of probability, called quantum logic or quantum probability. This quantum probability can be used to efficiently reflect "changes in perspective or state of mind" of the respondents as a result of the incorporation of a moral attribute in stated preference choice tasks. In 2020, a quantum choice model was introduced as a flexible new approach for understanding moral decision making in the field of transportation research. ${ }^{6}$ Given the success of using the quantum choice model in cognitive psychology and transportation, 1 possible application is to use it to address health equity.

This concept of a quantum choice model was adapted from Hancock et al.6,8 Conceptually, a participant would consider a choice task containing 2 alternatives. Under the quantum probability theory, the participant starts with a belief state. At this belief state, the participant may either have some underlying preference in favor of 1 alternative or feel indifferent between the 2 alternatives. When a decision is made, the belief state is projected to the chosen alternative. The alternative that has the projected vector 
with a larger amplitude represents a higher probability of being chosen. However, when an equity attribute is added, it can impact the participant's choice by moving the participant's initial belief state (quantum rotation) to either "ethical answerability belief state" or "not ethical answerability belief state". As a result, the participant would start from this new state (changes in perspective or state of mind) instead of their initial belief state. Subsequently, the probabilities for choosing these 2 alternatives are altered. In other words, the quantum model can mathematically capture a change in perspective through a quantum rotation. Therefore, conceptually, the choice model is improved or fits differently when the equity attributes are considered. From this model, patients' preferences to contribute to value assessment under the realization of health equity are captured. Finally, the value of each attribute derived from the 2 sets of DCE choice tasks with and without equity attributes can be compared and used to reflect the value of equity.

For example, this approach can be used to estimate social value for health gains from treatments. Two DCE surveys are designed with and without equity attributes such as health gain for individuals from an underrepresented population. Table 2 shows an example of the results indicating the observed choice share for an alternative $\mathrm{A}$ and the results from choice models. If the equity attribute is considered and the observed choice share of the alternative consistently change (eg, higher in this specific example), the results from the quantum choice model with rotation fits the data better due to its flexibility, judging from the higher average probability of chosen alternative A when including equity attributes.

On the other hand, the basic model could provide the opposite results. Similar to the first approach, how equity enters the preferences of the participants can be examined by modifying the equity attributes, for instance, focusing on equity in overall well-being or specifically on equity in health.

\section{Conclusions}

This paper describes 2 novel approaches, including latent class and quantum models, to rigorously value equity in health care. The latent class model is based on RUT with the classical theories of probability that has been widely used in choice modeling, while the quantum choice model is flexible enough to capture complex decisions as such decisions under equity. These approaches will improve existing value assessment methods that inadequately address health disparities and underrepresented populations.

\section{DISCLOSURES}

This study received no outside funding. Ngorsuraches has received research grants from Bristol Myers Squibb and through the University of Utah and PhRMA Foundation.

\section{REFERENCES}

1. Lakdawalla DN, Doshi JA, Garrison Jr LP, Phelps CE, Basu A, Danzon PM. Defining elements of value in health care-a health economics approach: an ISPOR Special Task Force Report. Value Health. 2018;21:131-39.

2. Memorial Sloan Kettering Cancer Center. DrugAbacus FAQs. Accessed December 1, 2016. https://www.drugpricinglab.org/tools/drug-abacus/

3. Cookson R, Mirelman AJ, Griffin S, et al. Using cost-effectiveness analysis to address health equity concerns. Value Health. 2017;20:206-12.

4. Chorus CG. Models of moral decision making: literature review and research agenda for discrete choice analysis. J Choice Modelling. 2015;16:69-85.

5. Alfano M. An introduction. In: Moral Psychology. Polity Press; 2016:1-22.

6. Hancock TO, Broekaert J, Hess S, Choudhury CF. Quantum choice models: a flexible new approach for understanding moral decision-making. J Choice Modelling. 2020;37:100235.

7. DeScioli P, Massenkoff M, Shaw A, Petersen MB, Kurzban R. Equity or equality? Moral judgments follow the money. Proc R Soc B. 2014;281:20142112.

8. Hancock TO, Broekaert J, Hess S, Choudhury CF. Quantum probability: a new method for modelling travel behavior. Transportation Research Part B. 2020;139:165-98.

9. Bruza PD, Wang Z, Busemeyer JR. Quantum cognition: a new theoretical approach to psychology. Trends Cogn Sci. 2015;19:383-93. 